\title{
Immune consequences of penfluridol treatment associated with inhibition of glioblastoma tumor growth
}

\author{
Alok Ranjan ${ }^{1}$, Stephen Wright ${ }^{1,2}$ and Sanjay K. Srivastava ${ }^{1,3}$ \\ ${ }^{1}$ Department of Biomedical Sciences and Cancer Biology Center, Texas Tech University Health Sciences Center, Amarillo, TX \\ 79106, USA \\ ${ }^{2}$ Departments of Internal Medicine and Biomedical Sciences, Texas Tech University Health Sciences Center, Amarillo, TX \\ 79106, USA \\ ${ }^{3}$ Department of Immunotherapeutics and Biotechnology, Texas Tech University Health Sciences Center, Abilene, TX 79106, USA \\ Correspondence to: Sanjay K. Srivastava, email: sanjay.srivastava@ttuhsc.edu \\ Keywords: MDSC, glioblastoma, anti-psychotic drug, Treg, macrophages \\ Abbreviations: PBMC: peripheral blood mononuclear cells; MDSC: myeloid derived suppressor cells; GBM: Glioblastoma multiforme; \\ Treg: regulatory $T$ cells \\ Received: October 20, $2016 \quad$ Accepted: March 13, $2017 \quad$ Published: April 26, 2017 \\ Copyright: Ranjan et al. This is an open-access article distributed under the terms of the Creative Commons Attribution License \\ 3.0 (CC BY 3.0), which permits unrestricted use, distribution, and reproduction in any medium, provided the original author and \\ source are credited.
}

\section{ABSTRACT}

Glioblastoma is the most common and lethal brain tumor associated with only $12 \%$ median survival rate of patients. Despite the development of advanced surgical, radiation or use of combinations of anti-cancer drugs, treatment for glioblastoma patients is still a challenge. The major contributing factor in glioblastoma progression and resistive nature is its ability to evade the immune surveillance. Hence, modulating the immune system in glioblastoma tumors could be an important strategy for anticancer therapeutics. Penfluridol, an antipsychotic drug has been shown to have anti-cancer properties in our recently published studies. The present study evaluates the immune response of penfluridol in glioblastoma tumors. Our results demonstrated that penfluridol treatment significantly suppressed glioblastoma tumor growth. Our current results demonstrated about $\mathbf{7 2} \%$ suppression of myeloid derived suppressor cells (MDSCs) with penfluridol treatment in mouse bearing U87MG glioblastoma tumors. MDSCs are known to increase regulatory $T$ cells (Treg), which are immunosuppressive in nature and suppresses M1 macrophages that are tumor suppressive in nature. Our results also showed suppression of regulatory $\mathrm{T}$ cells as well as elevation of M1 macrophages with penfluridol treatment by $58 \%$ and $57 \%$ respectively. Decrease in CCL4 as well as IFNy with penfluridol treatment was also observed indicating decrease in overall tumor inflammation. This is the first report demonstrating immune modulations by penfluridol treatment associated with glioblastoma tumor growth suppression prompting further investigation to establish penfluridol as a treatment option for glioblastoma patients.

\section{INTRODUCTION}

Glioblastoma (GBM) is a tumor that arises from astrocytes [1]. Astrocytes are star shaped cells and are known to support brain tissue. Glioblastoma tumors are highly malignant and aggressive in nature because cells divide quickly and are supported by a large network of blood vessels providing nutrients [1]. Malignant glioblastoma is highly infiltrated by myeloid-derived suppressor cells (MDSCs), which play a critical role in glioblastoma tumor progression and metastasis [2-4]. MDSCs are a heterogeneous group of cells and are a major component of tumor microenvironment. MDSCs, generated in bone marrow and in host bearing tumors, migrate to lymphoid organs and tumors, and support the formation of tumor microenvironment [5]. Acquired resistance to chemotherapy is difficult to overcome during cancer treatment. Induction and expansion of MDSCs play a critical role in chemoresistance [6]. Currently, glioblastoma is 
difficult to manage by conventional chemotherapies. Hence, new treatment option for glioblastoma is indispensable.

Like MDSCs, regulatory T cells (Treg) are also a part of immunosuppressive tumor microenvironment [7]. The association of regulatory $\mathrm{T}$ cells in overall poor prognosis of several neoplasms has been established [8]. In addition, recent studies demonstrated that MDSCs can also induce regulatory T cells [9]. On the other hand, macrophages have critical role in cancer progression, and play a key role in inflammation and cancer [10]. Tumor-associated macrophages facilitate neoplastic transformation as well as promote signaling cascades for metastasis [11]. MDSCs and macrophages are present in most solid tumors and play a critical role in overall inflammation and immunosuppressive activity [12]. Cross-talk between MDSCs and macrophages promote tumor prognosis by affecting antitumor immunity [12]. The resulting chronic inflammation can predispose to cancer [13]. Several anti-inflammatory drugs have been shown to have an anti-cancer effect [14]. Interestingly, Baune et.al has demonstrated in a study that antipsychotic drugs have an anti-inflammatory effect [15].

Penfluridol, an antipsychotic drug has been demonstrated by us to inhibit the growth of metastatic breast tumors in brain [16]. In another study, we observed that penfluridol significantly suppresses the growth of various glioblastoma tumor cells in vitro and in vivo [17]. In the current study, we evaluated immune consequences of penfluridol treatment associated with inhibiting glioblastoma tumor growth.

Our results show substantial growth suppression of glioblastoma tumors and MDSC levels by penfluridol treatment. In addition, penfluridol treatment reduced Tregs and enhanced M1 macrophages. This is the first report of immune modulation by penfluridol treatment associated with suppression of glioblastoma tumor growth.

\section{RESULTS}

\section{Penfluridol inhibits glioblastoma tumor growth}

To determine the efficacy of penfluridol in inhibiting the growth of glioblastoma, U87MG glioblastoma cells were implanted subcutaneously on both flanks of athymic nude mice. Once each mouse attained about $70-100 \mathrm{~mm}^{3}$ tumors, mice were randomly divided into two groups. Control mice received vehicle only whereas the treatment group of mice received $10 \mathrm{mg} / \mathrm{kg}$ penfluridol everyday by oral gavage. The experiment was terminated at day 48 due to excessive tumor burden in control mice. After 48 days of penfluridol treatment, glioblastoma tumor growth was suppressed by $65 \%$ (Figure 1A-1C).

\section{Reduction in myeloid-derived suppressor cells with penfluridol treatment}

Myeloid-derived suppressor cells (MDSCs) are known to promote growth of tumors. High infiltrations of MDSCs are also known to regulate stem cells like characteristics in tumors. We used CD11b and Gr1 markers to analyze mouse MDSCs after treatment of mice with $10 \mathrm{mg} / \mathrm{kg}$ penfluridol by oral gavage everyday till day 48. Blood from control and penfluridol treated mice was collected and PBMCs were separated. MDSCs were analyzed by gating on forward and side scattered profile of the cells using flow cytometer. We observed a $72 \%$ reduction of mouse MDSCs with penfluridol treatment (Figure 1D). This observation indicated that penfluridol treatment suppressed MDSCs, which play a significant role in promoting tumor growth.

\section{Modulation in spleen weight with penfluridol treatment}

The spleen is a peripheral immune organ and plays a critical role in regulating the immune system of the body. Shrinkage of spleen as well as inhibition of spleen cell proliferation has been observed in late stage tumors where the immune system is suppressed [18, 19]. Our results showed that the average weight of spleens in mice treated with penfluridol was modestly increased as compared to the spleens from control mice (Figure 2A-2B), indicating that penfluridol may have increased splenic cells proliferation, which correlate with inhibition of tumor growth associated with immune system.

\section{Suppression of regulatory $T$ cells by penfluridol treatment}

Translation of mouse data to human fails due to significant differences in mouse and human immune systems. In order to confirm effects of penfluridol treatment on human immune system, we injected 40 x $10^{6}$ human PBMCs (isolated from human blood) intraperitoneally in SCID-NOD mice. A week after human PBMC injection, each mouse was injected with 1 x $10^{6}$ U87MG glioblastoma cells subcutaneously. Penfluridol $10 \mathrm{mg} / \mathrm{kg}$ was administered to mice everyday by oral gavage once tumor volume reached around $70-100 \mathrm{~mm}^{3}$, whereas the control group of mice received vehicle only. The experiment was terminated at day 40 due to excessive tumor burden in the control group of mice. After termination of the experiment, PBMCs were collected from the blood of control and penfluridol treated mice and double stained with FoxP3 and CD4 antibodies for regulatory $\mathrm{T}$ cells (Treg). Our results show that penfluridol treatment reduced regulatory $\mathrm{T}$ cells (Treg) by $58 \%$, indicating an increase in immune surveillance in mice bearing human tumors (Figure 3).

\section{Elevation of M1 macrophages with penfluridol treatment in the tumor microenvironment}

Tumors from control and penfluridol treated mice were aseptically removed after terminating the 
experiment at day 40. Tumors were suspended into single cell suspension using a tumor dissociation kit and gentle MACS Dissociator. Single cell suspension from control and penfluridol treated tumors were processed and analyzed after double staining with CD86 and IL12 antibodies for antigens present on M1 macrophages. M1 macrophages are known to be involved in tumor growth suppression [20]. Our results demonstrated that penfluridol treatment resulted in a 57\% increase of M1 macrophages as compared to control tumors (Figure 4A). Interestingly, we did not observe any change in the cells stained with CD88 expression, a marker of macrophages [21].

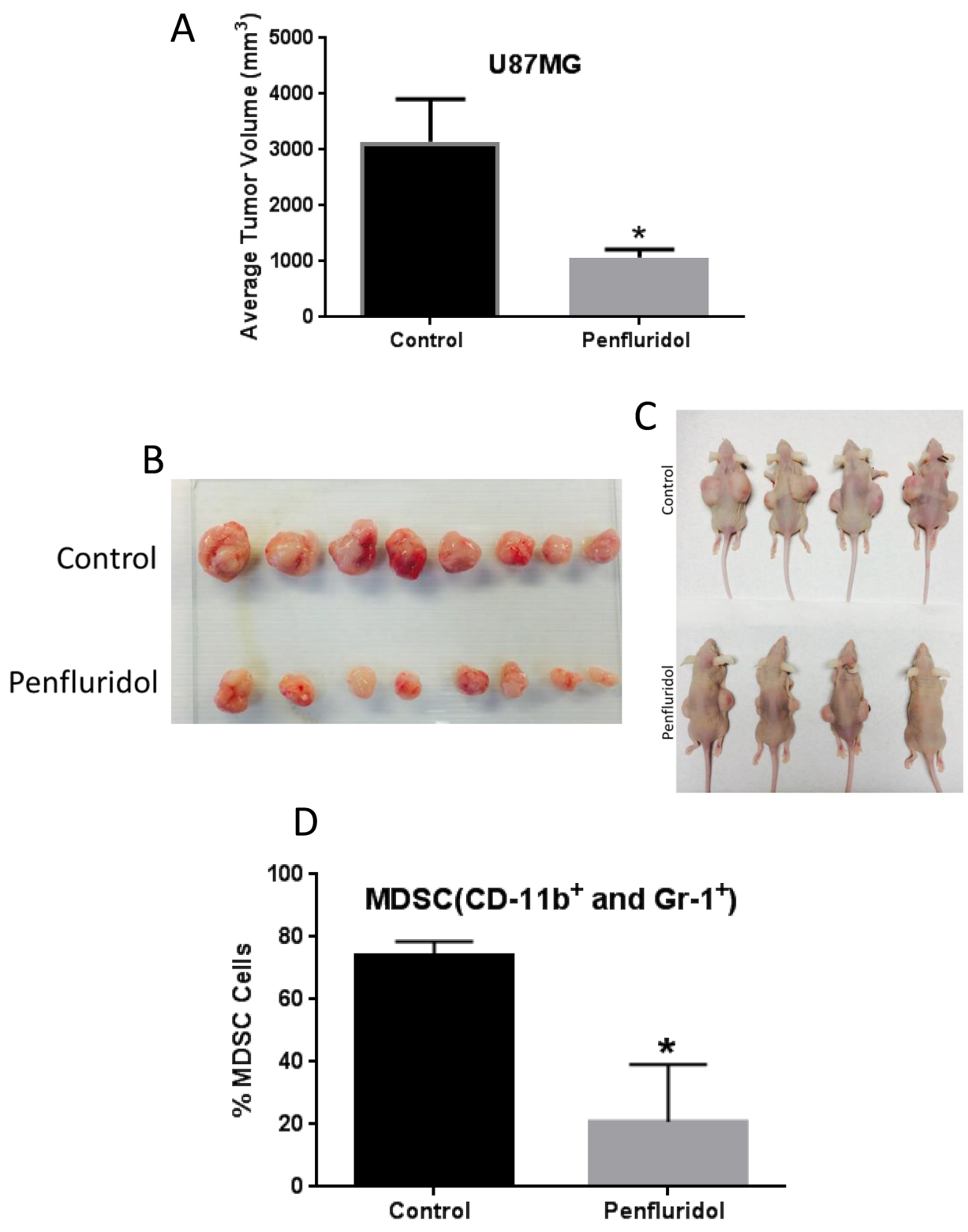

Figure 1: Penfluridol suppresses glioblastoma tumor growth and associated MDSCs. $1 \times 10^{6}$ U $87 \mathrm{MG}$ cells in $1: 1 \mathrm{mixture}$ of PBS and matrigel were implanted in right and left flanks of 4-6 week old athymic nude mice. Treatment with $10 \mathrm{mg} / \mathrm{kg}$ penfluridol by oral gavage everyday started once tumor size was $70-100 \mathrm{~mm}^{3}$ after tumor cells injection till day 48 . Tumors from all control and penfluridol treated mice were removed at day 48. (A) Bar graph representing average tumor volume of control and penfluridol treated mice at day 48. Values were plotted as mean \pm SEM. (B-C) Representative images of tumors as well as mice bearing tumors from control and treated group. Peripheral blood mononuclear cells (PBMCs) were collected from the blood obtained from control and penfluridol treated U87MG tumor-bearing athymic nude mice. Modulation of $\mathrm{CD} 11 \mathrm{~b}^{+}$and $\mathrm{Gr}-1^{+}$cells was analyzed by immunostaining and fluorescence cytometry to determine the effects of penfluridol on mouse myeloid derived suppressor cells (MDSCs); statistical analysis was performed by Student's $\mathrm{t}$ test. (D) Bar chart showing percent MDSCs which were double stained with CD11b and Gr-1 mouse antibodies. *Statistically significant at $\mathrm{p}<0.05$ when compared with control. 


\section{Penfluridol suppressed tumor inflammation}

As chronic inflammation plays a significant role in tumor progression, we decided to analyze inflammatory markers in the tumor microenvironment after penfluridol treatment. Subcutaneously implanted U87MG tumors from SCID-NOD mice injected with human PBMCs were examined for inflammatory markers after terminating the experiment. Tumors obtained from control and penfluridol treated mice were lysed and ELISA assay was performed for IFN $\gamma$ and CCL4. IFN $\gamma$ and CCL4 are known to play critical roles in tumor progression
$[22,23]$. Our results demonstrated that penfluridol treatment resulted in $54 \%$ and $49 \%$ reduction of CCL4 and IFN $\gamma$ respectively, indicating suppression of inflammation in the tumor microenvironment (Figure 5A-5B).

\section{DISCUSSION}

Glioblastoma is one of the deadliest cancers. Currently, radiation and chemotherapy are the available treatments for glioblastoma, however, glioblastoma cells are radioresistant and chemoresistant leading
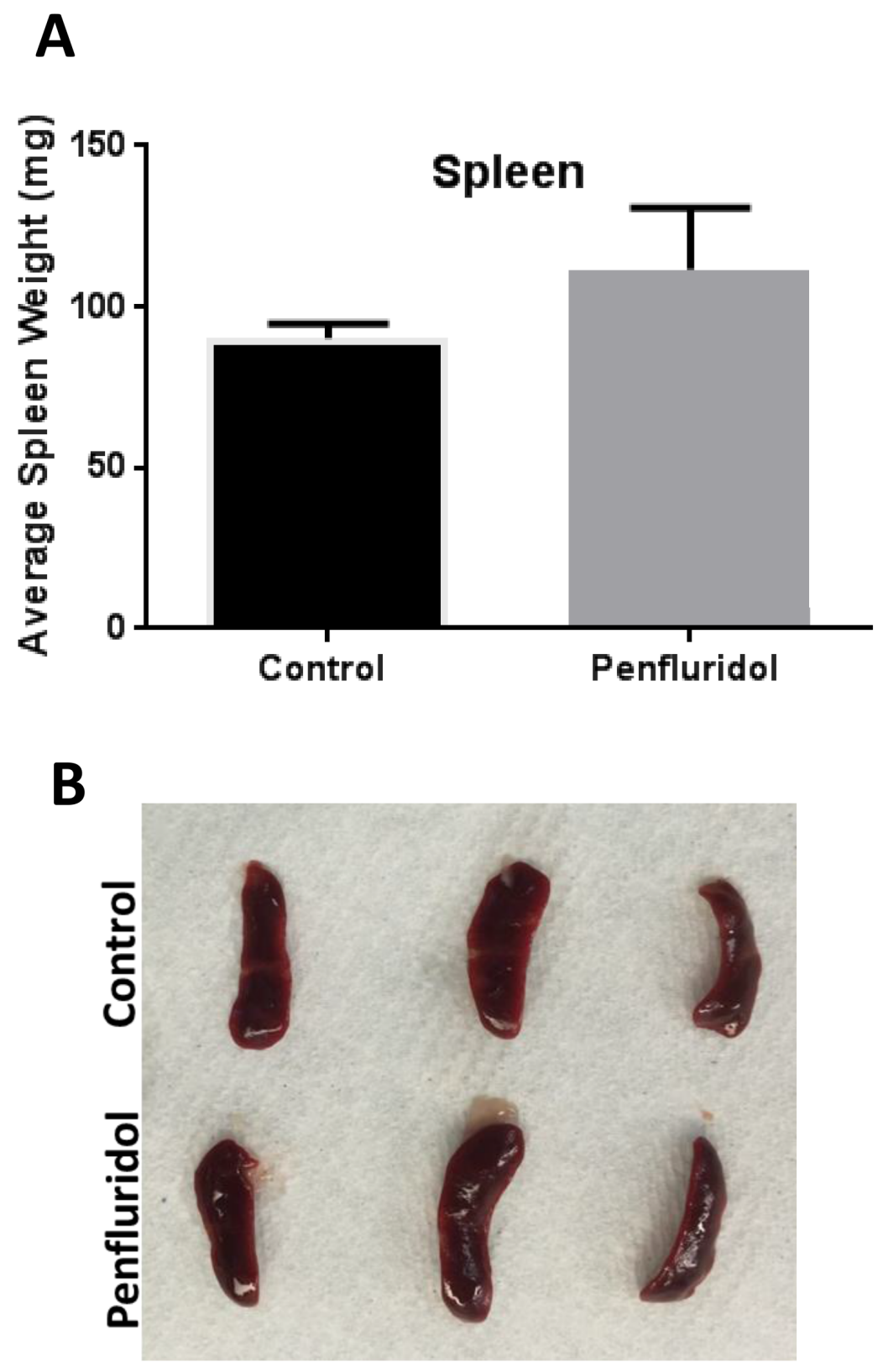

Figure 2: Increase in spleen weight with penfluridol treatment: Spleens from control and penfluridol treated mice were removed and weights of spleens were taken. (A) Bar chart showing average weight of spleen in control and penfluridol treated group. Values were plotted as mean \pm SEM. (B) Image of spleen from control and penfluridol treated mice. 
to reoccurrence after conventional therapy [24]. The immune system's natural ability to recognize and kill abnormal cells in the body may prevent development of cancer. However, cancer cells evade immune response by inducing several immune cells that can suppress natural defense of the body [25]. Tumor progression has long been associated with chronic inflammatory microenvironment and infiltration of MDSCs [26]. MDSCs infiltration of the tumor microenvironment has also been associated with glioblastoma stem cells characteristics [27]. In addition, glioblastoma progression is connected with immunosuppression driven by elevation in Treg and MDSCs in the tumor microenvironment [26]. Elevated Treg in peripheral blood and tumor microenvironment has been associated with tumor progression. Temozolomide, an FDA approved drug against glioblastoma has been known to reduce circulating Treg further increasing the activity of cytotoxic T cells against tumors [28, 29]. Furthermore, macrophages are known to be associated with MDSCs and involved in inflammation and immunosuppressive effects in the tumor microenvironment [12]. Overall, glioblastoma tumors create an immunosuppressive environment and engage several pathways to evade innate immune surveillance.

In our previous study, we demonstrated that penfluridol treatment significantly suppressed the growth of metastatic breast tumors in brain [16]. Our results from another study showed that $10 \mathrm{mg} / \mathrm{kg}$ penfluridol everyday by oral gavage suppressed the growth of glioblastoma tumors in a subcutaneous and intracranial model [17]. In the current study, we evaluated immune modulations after treatment with penfluridol. Several lines of evidence have suggested that chronic inflammation predisposes to cancer [13]. Our results demonstrated that oral administration of $10 \mathrm{mg} / \mathrm{kg}$ penfluridol everyday for 48 days reduced mouse MDSCs by $72 \%$. Generally, chemotherapy is associated with immune suppression in the patients. Polysaccharides, such as ginseng, when used in combination with anti-cancer drugs was shown to improve drug induced immunosuppression [30]. Beneficial effect of polysaccharide in combination with anti-cancer drugs was associated with increase in spleen weight and stimulation of lymphocyte proliferation [30]. Similarly to these studies, we also observed a minor increase in spleen weight with penfluridol treatment indicating increased splenic activity and immune stimulation, which may have led to enhanced immune surveillance to inhibit tumor growth.

Different animal models are used to mimic human disease, however, in most of the cases, translation of mouse data to human fails due to significant differences between mouse and human immune systems [31]. We therefore evaluated the effect of penfluridol in glioblastoma tumors by injecting human PBMCs in SCIDNOD mice prior to human tumor implantation. MDSCs inhibit adaptive immunity of the body by inducing Treg cells [32]. Treg cells are $\mathrm{CD}^{+} \mathrm{T}$ cells with elevated FOXP3 and are known to suppress innate immune

\section{Regulatory Tcells (FoxP3 and CD4)}

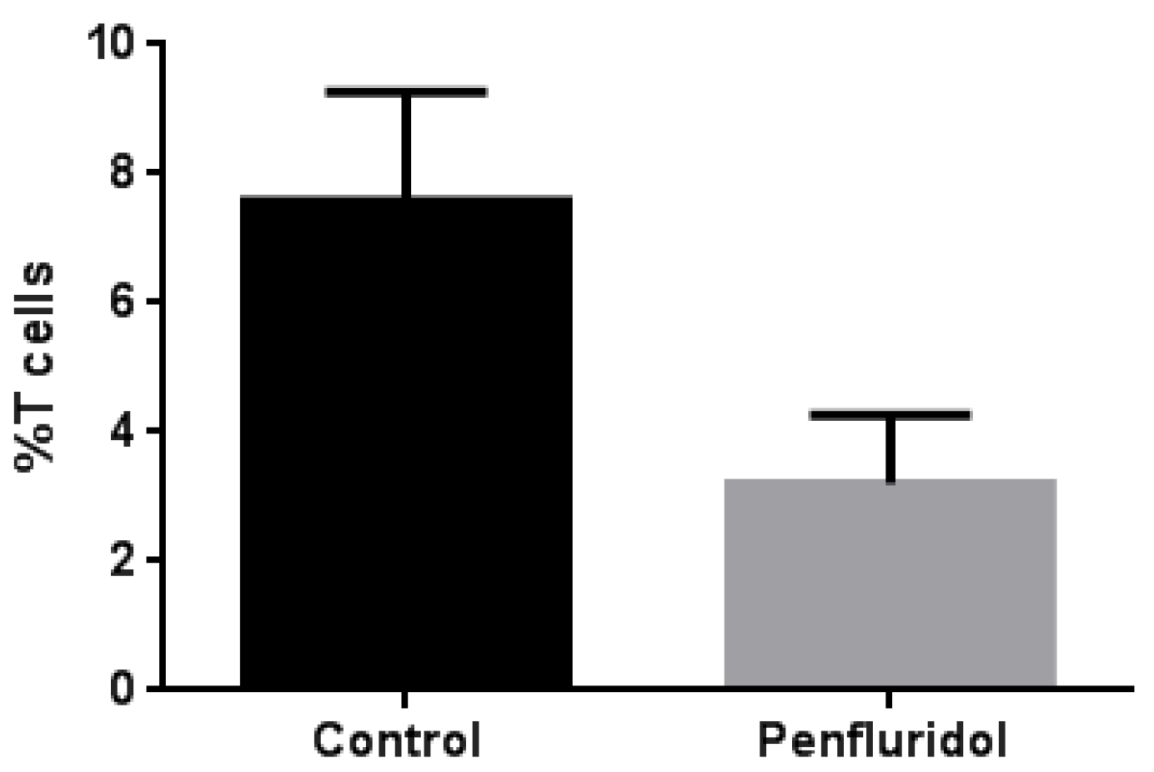

Figure 3: Penfluridol suppresses regulatory T cells (Treg). About $40 \times 10^{6}$ Human peripheral blood mononuclear cells (PBMCs) cells were injected i.p. in $100 \mu \mathrm{L}$ PBS in SCID-NOD mice. The treatment group received $10 \mathrm{mg} / \mathrm{kg}$ penfluridol by oral gavage everyday; control mice received vehicle alone. Modulation of regulatory T (Treg) cells was monitored by immunostaining and fluorescence cytometry to determine the effects of penfluridol. Values were plotted as mean \pm SEM. Statistical analysis was performed by Student's $t$ test. 
responses [33]. Our results showed that penfluridol treatment by oral gavage reduced Treg cells in SCIDNOD mouse injected with human PBMCs. Macrophages, primarily M1 and M2, subtypes are a major source of inflammatory cytokines. M1 macrophages secrete interleukin 12 (IL-12) that helps in generation of T helper 1 (TH-1) adaptive immunity to directly induce cytotoxic effects in tumor cells [34]. M2 macrophages are immune suppressive in nature, contribute to matrix remodeling and hence support tumor growth [35]. M1 macrophages are characterized by high expression of CD86 and IL-12 [36]. In our study, we observed that tumor growth suppression by penfluridol treatment was associated with increased M1 macrophages, which may have led to induction of cytotoxic effect in tumor cells.

Inflammation has long been associated with tumor growth [37]. It plays critical role in modulating immune surveillance and hence altered response to

\section{A Tumor M1 Macrophages (CD86 + IL12)}
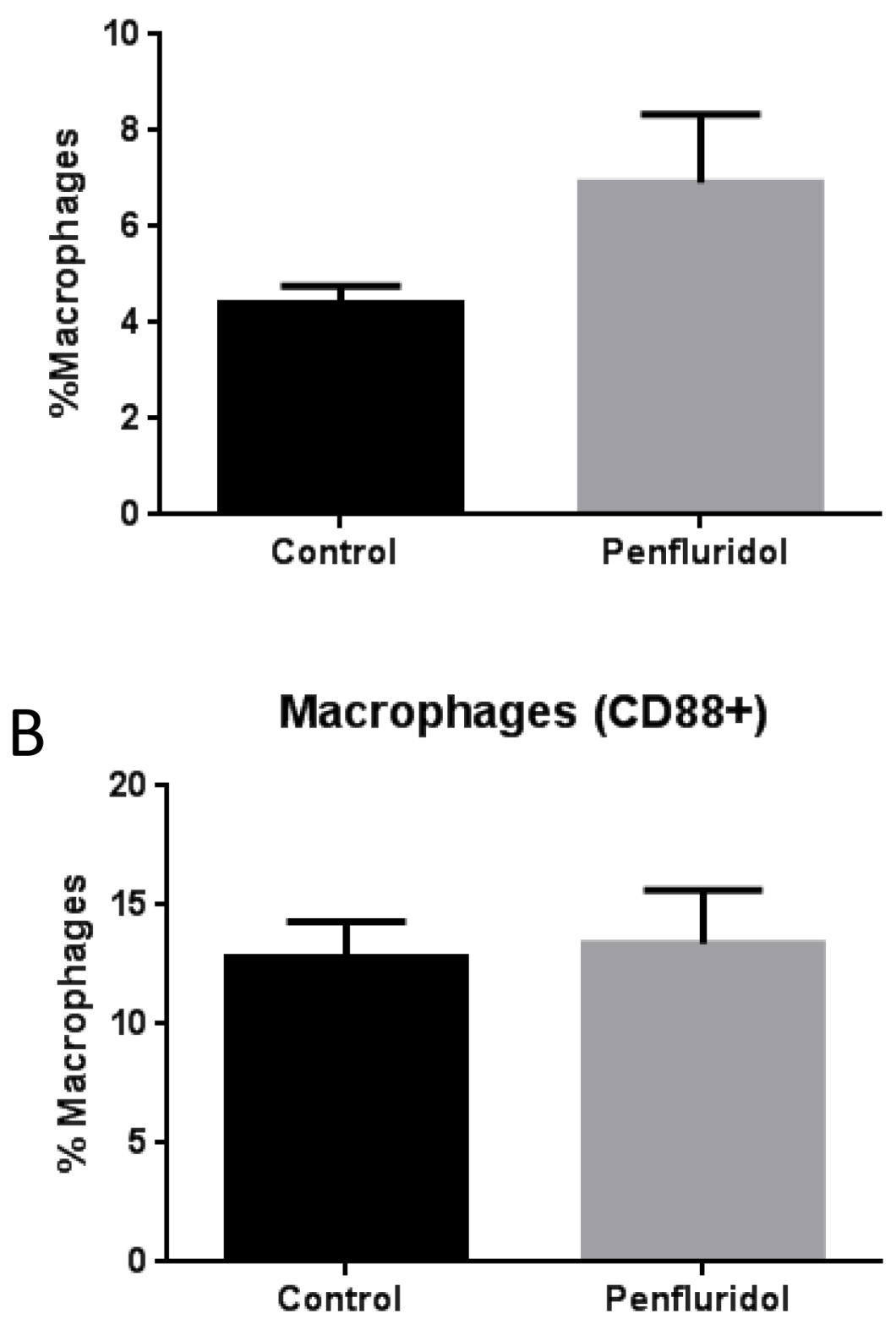

Figure 4: Elevation of M1 macrophages in tumor with penfluridol treatment. Subcutaneously implanted U87MG glioblastoma tumors in SCID-NOD mice were removed from control and penfluridol treated mice at day 40. Tumors were processed and suspended into single cell suspension with the help of tumor dissociator kit and use of gentle MACS dissociator. Effect of penfluridol treatment on macrophages was analyzed by immunostaining and florescence cytometry. (A) Bar graph representing percent monocytic cells double stained with CD86 and IL12 human specific antibodies. (B) Percent cells which were single stained with CD88 human specific antibody. 
therapy. Infiltrating macrophages have been established as the critical component of inflammation during tumor progression. It has also been established that CCL4 plays a crucial role in macrophage-mediated tumorigenic signaling [22]. In our current study, we observed that penfluridol treatment reduced infiltrating human CCL4 in the tumor microenvironment by $54 \%$ in SCID-NOD mice injected with human PBMCs. Under certain conditions,
IFN $\gamma$ plays critical role in anti-tumor host immunity and supports tumor growth [23]. In a few clinical trials, IFN $\gamma$ treatment negatively affected patients outcome whereas in other trials, it provided positive effect in cancer patients [23]. Thus IFN $\gamma$ has been established to have dual role: one as a hallmark of anti-tumor immunity and another to support tumor growth by immune escape phenomena, a mechanism mediated through PD-L-1 expression [23].
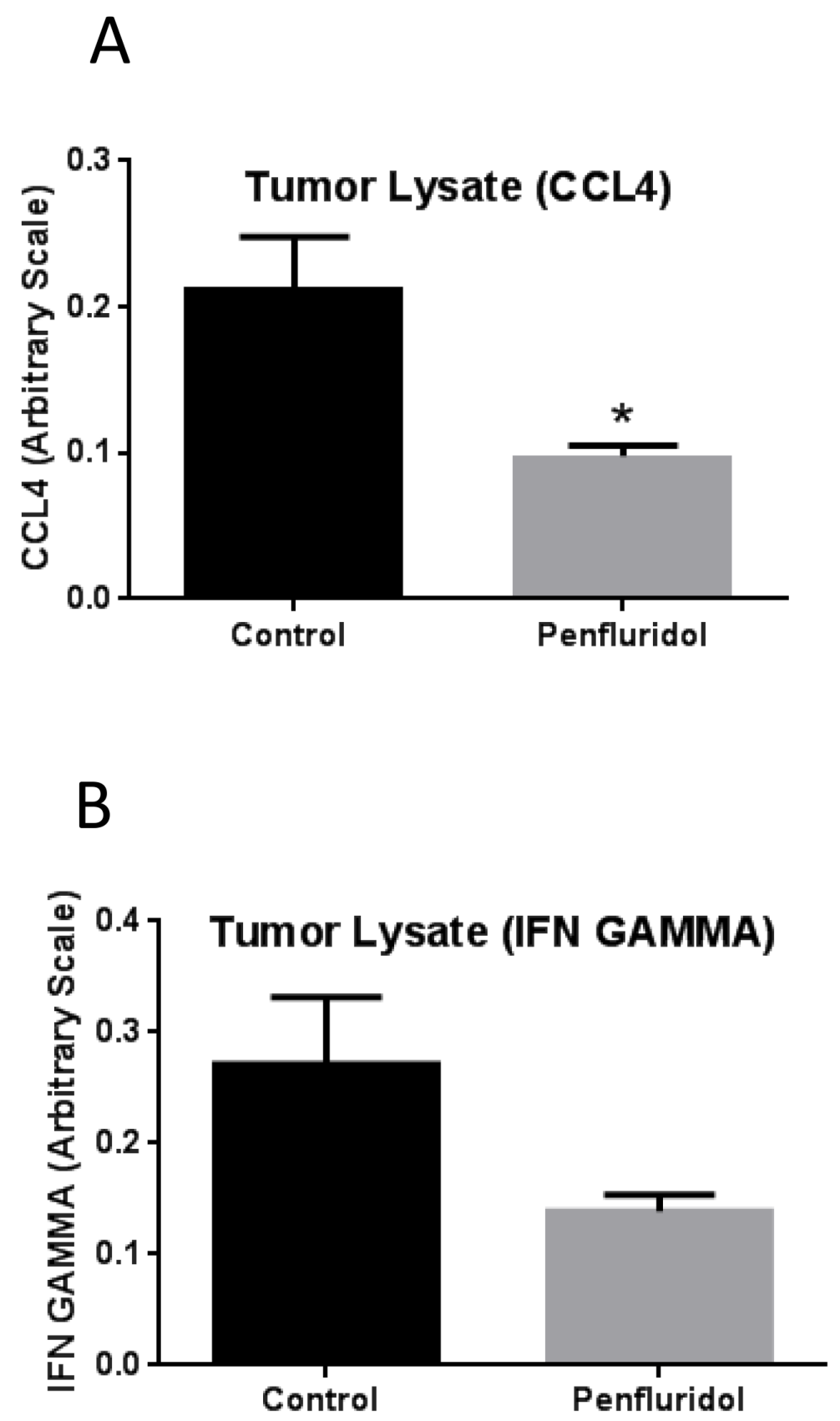

Figure 5: Suppression of tumor inflammation with penfluridol treatment. After removing U87MG tumors, a part of the tumors were lysed and protein concentration was estimated in different control and penfluridol treated tumor lysate. Equal amount of protein was

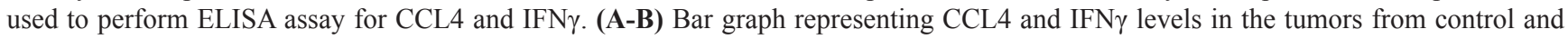
treatment group. Values were plotted as mean \pm SEM. *Statistically significant at $\mathrm{p}<0.05$ when compared with control. 
Interestingly, we observed that penfluridol significantly suppressed human IFN $\gamma$ in the tumor microenvironment in SCID-NOD mice injected with human PBMCs and implanted with human U87MG glioblastoma tumors.

In summary, suppression of GBM tumor growth correlated with reduction of immune suppressive cells (Treg and MDSC), an increase of tumor killing macrophages (M1), and reduction of markers of chronic inflammation (CCL4 and IFN $\gamma$ ) in the tumor microenvironment. We suggest that immune enhancement may have been involved in inhibition of tumor growth of glioblastoma. This is the first report, demonstrating immune modulation with penfluridol treatment associated with glioblastoma tumor growth inhibition. An imbalance in the immune system during tumor progression is associated with chemoresistant tumors and our study lays the foundation of effective treatment for glioblastoma by immune modulation. Our study further supports future clinical development of immunotherapies to suppress glioblastoma and preclinical investigation of combining temozolomide and penfluridol and other immune modulatory agents to attempt to eliminate glioblastoma.

\section{MATERIALS AND METHODS}

\section{Ethics statement}

Experiments in mice were conducted in accordance with the ethical standards and according to approved protocol by the Institutional Animal Care and Use Committee (IACUC), Texas Tech University Health Sciences Center, Amarillo, Texas.

\section{Cell culture}

U87MG glioblastoma cells used in this study were purchased from ATCC and maintained in EMEM supplemented with $10 \%$ FBS and $1 \%$ PSN. Cells used in the experiments were within twenty passages after receipts or resuscitation.

\section{U87MG subcutaneous implantation}

4-6 week old female athymic nude mice were purchased from Harlan Laboratories (Livermore, CA). IACUC approved the use of athymic nude mice. All the experiments were performed under the strict compliance and regulations. As described by us previously, $100 \mu \mathrm{L}$ cell suspension containing $1 \times 10^{6} \mathrm{U} 87 \mathrm{MG}$ cells in $1: 1$ mixture of PBS and matrigel were implanted in both flanks of mice. Mice were divided into two groups with 5 mice in each group once tumor volume reached around 70-100 $\mathrm{mm}^{3}$. Group I served as control and received the vehicle only whereas group II received $10 \mathrm{mg} / \mathrm{kg} /$ day penfluridol by oral gavage. Penfluridol was purchased from SigmaAldrich and stock of penfluridol was made in DMSO which was further diluted in water/PEG300/ethanol/2\% acetic acid in 8:3:0.13:1 v/v [38]. Tumor volume was measured twice a week till day 48 by using vernier caliper and as described by us before [16, 39]. At day 48, mice were humanely sacrificed and tumors were removed. The results of this experiment have been published by us recently [17]. A part of the tumor from this experiment was used for current study.

\section{PBMC collection from mouse blood and flow cytometric analysis}

To evaluate the effect of penfluridol treatment on immune cells, blood was collected from control and treatment group. The blood collected from each mouse by cardiac puncture was diluted 10 fold with $\mathrm{RBC}$ lysis buffer and incubated for 15 minutes at room temperature on a shaker. RBC lysis step was repeated in case complete lysis not achieved. After lysis of RBCs, cells were washed with PBS followed by a second washing with FACS buffer $(2 \%$ heat inactivated fetal bovine serum and $2 \mathrm{mM}$ ethylenediaminetetraacetic acid (EDTA) in PBS) as described before by us [40]. PBMC from each mouse were equally divided into two groups. Each set of cells was re-suspended in an equal volume of FACS buffer. CD11b Gr-1 FoxP3, CD4, CD86, IL12 and CD88 antibodies were purchased from Miltenyi Biotech. The samples were first blocked using blocking reagent for 15 minutes at $4^{\circ} \mathrm{C}$ in dark. After blocking, cells were washed and suspended in FACS buffer. After suspension in FACS buffer, appropriate antibodies were added and samples were incubated for 30 minutes on ice in dark. The samples were then washed and re-suspended in $300 \mu \mathrm{L}$ of FACS buffer. Accuri C6 flow cytometer software was used to analyze the effect of penfluridol treatment on MDSCs, regulatory $\mathrm{T}$ cells (Treg) and macrophages.

\section{Human PBMC isolation from buffy coat}

Healthy human buffy cones from anonymous donors were obtained from Coffee Memorial Blood Bank, Amarillo, TX. Samples from each buffy cone were diluted with PBS to make final volume of $50 \mathrm{ml}$. Diluted samples were divided into two tubes with $25 \mathrm{ml}$ each, after which, $15 \mathrm{ml}$ PBS was further added. Samples were gently layered over 10-15ml Ficoll-Paque reagent (GE Lifesciences) as described by us before [40]. Samples were gently centrifuged for 40 minutes at 400 $\mathrm{x} g$ maintaining $20^{\circ} \mathrm{C}$ temperature and without applying any brakes. A clear band of PBMCs was observed at the interface of Ficoll and plasma, and this band was collected. The collected PBMCs were washed with PBS and incubated at $37^{\circ} \mathrm{C}$ with red blood cell (RBC) lysis buffer. The cells were then centrifuged, washed with PBS and collected. 


\section{Injection of PBMC in SCID mice}

4-6 week old female SCID-NOD mice were obtained from Harlan laboratories and maintained under specific pathogen free condition. After a week of acclimatization in the new environment, $100 \mu 1$ PBS containing $40 \times 10^{6}$ PBMCs were injected by intraperitoneal route in each mouse. After seven days of PBMCs injection, about $1 \times 10^{6} \mathrm{U} 87 \mathrm{MG}$ cells in 1:1 mixture of PBS and matrigel were injected subcutaneously in both the flanks of SCIDNOD mice. Once tumor volume was $50-70 \mathrm{~mm}^{3}$, mice were treated with $10 \mathrm{mg} / \mathrm{kg}$ penfluridol by oral gavage everyday with 5 mice each in control and treatment group respectively.

\section{ELISA assay using tumor lysate}

After terminating the experiment at day 40, tumors from SCID-NOD mice were removed from control and penfluridol treated groups. Tumors were lysed using protease and phosphatase inhibitors in PBS with subsequent vortexing and sonication. Protein was estimated using Bradford reagent (Bio-Rad). Equal amounts of protein from control and penfluridol treated tumor lysate samples were used to perform ELISA assay for CCL4 and IFN $\gamma$ following the manufacturer's instructions (Affymetrix, ebioscience).

\section{Statistical analysis}

Statistical analysis was performed using Prism 6.0 (GraphPad software Inc.). Tumor results were represented as means \pm SEM. Data was analyzed by Student's t-test. Differences were considered statistically significant at $\mathrm{p}<0.05$ when compared with control.

\section{CONFLICTS OF INTEREST}

The authors declare no conflicts of interest.

\section{GRANT SUPPORT}

Supported in part by R01 grant CA129038 (to S.K.S) awarded by the National Cancer Institute, NIH.

\section{REFERENCES}

1. Zong H, Verhaak RG, Canoll P. The cellular origin for malignant glioma and prospects for clinical advancements. Expert Rev Mol Diagn. 2012; 12:383-394.

2. Kohanbash G, Okada H. Myeloid-derived suppressor cells (MDSCs) in gliomas and glioma-development. Immunol Invest. 2012; 41:658-679.

3. Raychaudhuri B, Rayman P, Ireland J, Ko J, Rini B, Borden EC, Garcia J, Vogelbaum MA, Finke J. Myeloid-derived suppressor cell accumulation and function in patients with newly diagnosed glioblastoma. Neuro Oncol. 2011; 13:591-599.

4. Thaci B, Ahmed AU, Ulasov IV, Wainwright DA, Nigam P, Auffinger B, Tobias AL, Han Y, Zhang L, Moon KS, Lesniak MS. Depletion of myeloid-derived suppressor cells during interleukin-12 immunogene therapy does not confer a survival advantage in experimental malignant glioma. Cancer Gene Ther. 2014; 21:38-44.

5. Kumar V, Patel S, Tcyganov E, Gabrilovich DI. The nature of myeloid-derived suppressor cells in the tumor microenvironment. Trends Immunol. 2016; 37:208-220.

6. Deng Z, Rong Y, Teng Y, Zhuang X, Samykutty A, Mu J, Zhang L, Cao P, Yan J, Miller D, Zhang HG. Exosomes miR-126a released from MDSC induced by DOX treatment promotes lung metastasis. Oncogene. 2017; 36:639-651.

7. Gorgun GT, Whitehill G, Anderson JL, Hideshima T, Maguire C, Laubach J, Raje N, Munshi NC, Richardson PG, Anderson KC. Tumor-promoting immune-suppressive myeloid-derived suppressor cells in the multiple myeloma microenvironment in humans. Blood. 2013; 121:2975-2987.

8. Whiteside TL. Immune responses to malignancies. J Allergy Clin Immunol. 2010; 125:S272-S283.

9. Yang R, Cai Z, Zhang Y, Yutzy WH, Roby KF, Roden RB. CD80 in immune suppression by mouse ovarian carcinomaassociated Gr-1+CD11b+ myeloid cells. Cancer Res. 2006; 66:6807-6815.

10. Conway EM, Pikor LA, Kung SH, Hamilton MJ, Lam S, Lam WL, Bennewith KL. Macrophages, inflammation, and lung cancer. Am J Respir Crit Care Med. 2016; 193:116-130.

11. Williams CB, Yeh ES, Soloff AC. Tumor-associated macrophages: unwitting accomplices in breast cancer malignancy. NPJ Breast Cancer. 2016; 2:15025.

12. Beury DW, Parker KH, Nyandjo M, Sinha P, Carter KA, Ostrand-Rosenberg S. Cross-talk among myeloid-derived suppressor cells, macrophages, and tumor cells impacts the inflammatory milieu of solid tumors. J Leukoc Biol. 2014; 96:1109-1118.

13. Shacter E, Weitzman SA. Chronic inflammation and cancer. Oncology (Williston Park). 2002; 16:217-229.

14. Rayburn ER, Ezell SJ, Zhang R. Anti-inflammatory agents for cancer therapy. Mol Cell Pharmacol. 2009; 1:29-43.

15. Baune BT, Eyre H. Anti-inflammatory effects of antidepressant and atypical antipsychotic medication for the treatment of major depression and comorbid arthritis: a case report. J Med Case Rep. 2010; 4:6.

16. Ranjan A, Gupta P, Srivastava SK. Penfluridol: an antipsychotic agent suppresses metastatic tumor growth in triple-negative breast cancer by inhibiting integrin signaling axis. Cancer Res. 2016; 76:877-890.

17. Ranjan A, Srivastava SK. Penfluridol suppresses glioblastoma tumor growth by Akt-mediated inhibition of 
GLI1. Oncotarget. 2017; 8:32960-32976. doi: 10.18632/ oncotarget. 16515.

18. Prehn RT. The paradoxical effects of splenectomy on tumor growth. Theor Biol Med Model. 2006; 3:23.

19. Richards J, McNally B, Fang X, Caligiuri MA, Zheng P, Liu Y. Tumor growth decreases NK and B cells as well as common lymphoid progenitor. PLoS One. 2008; 3:e3180.

20. Yuan A, Hsiao YJ, Chen HY, Chen HW, Ho CC, Chen YY, Liu YC, Hong TH, Yu SL, Chen JJ, Yang PC. Opposite effects of M1 and M2 macrophage subtypes on lung cancer progression. Sci Rep. 2015; 5:14273.

21. Gasque P, Singhrao SK, Neal JW, Gotze O, Morgan BP. Expression of the receptor for complement C5a (CD88) is up-regulated on reactive astrocytes, microglia, and endothelial cells in the inflamed human central nervous system. Am J Pathol. 1997; 150:31-41.

22. Fang LY, Izumi K, Lai KP, Liang L, Li L, Miyamoto H, Lin WJ, Chang C. Infiltrating macrophages promote prostate tumorigenesis via modulating androgen receptor-mediated CCL4-STAT3 signaling. Cancer Res. 2013; 73:5633-5646.

23. Mandai M, Hamanishi J, Abiko K, Matsumura N, Baba $\mathrm{T}$, Konishi I. Dual faces of IFN $\gamma$ in cancer progression: a role of PD-L1 induction in the determination of pro- and antitumor immunity. Clin Cancer Res. 2016; 22:2329-2334.

24. Altaner C. Glioblastoma and stem cells. Neoplasma. 2008; 55:369-374.

25. Naidoo J, Page DB, Wolchok JD. Immune modulation for cancer therapy. Br J Cancer. 2014; 111:2214-2219.

26. Chang AL, Miska J, Wainwright DA, Dey M, Rivetta CV, Yu D, Kanojia D, Pituch KC, Qiao J, Pytel P, Han $\mathrm{Y}, \mathrm{Wu} \mathrm{M}$, Zhang L, et al. CCL2 produced by the glioma microenvironment is essential for the recruitment of regulatory $\mathrm{T}$ cells and myeloid-derived suppressor cells. Cancer Res. 2016; 76:5671-5682.

27. Otvos B, Silver DJ, Mulkearns-Hubert EE, Alvarado AG, Turaga SM, Sorensen MD, Rayman P, Flavahan WA, Hale JS, Stoltz K, Sinyuk M, Wu Q, Jarrar A, et al. Cancer stem cell-secreted macrophage migration inhibitory factor stimulates myeloid derived suppressor cell function and facilitates glioblastoma immune evasion. Stem Cells. 2016; 34:2026-2039.

28. Ridolfi L, Petrini M, Granato AM, Gentilcore G, Simeone E, Ascierto PA, Pancisi E, Ancarani V, Fiammenghi L, Guidoboni M, de Rosa F, Valmorri L, Scarpi E, et al. Low-dose temozolomide before dendritic-cell vaccination reduces (specifically) $\mathrm{CD} 4+\mathrm{CD} 25++$ Foxp $3+$ regulatory
T-cells in advanced melanoma patients. J Transl Med. 2013; $11: 135$.

29. Markl B, Paul B, Schaller T, Kretsinger H, Kriening B, Schenkirsch G. The role of lymph node size and FOXP3+ regulatory $\mathrm{T}$ cells in node-negative colon cancer. J Clin Pathol. 2017; 70:443-447.

30. Chen S, Wang Z, Huang Y, O'Barr SA, Wong RA, Yeung $\mathrm{S}$, Chow MS. Ginseng and anticancer drug combination to improve cancer chemotherapy: a critical review. Evid Based Complement Alternat Med. 2014; 2014:168940.

31. Zschaler J, Schlorke D, Arnhold J. Differences in innate immune response between man and mouse. Crit Rev Immunol. 2014; 34:433-454.

32. Ostrand-Rosenberg S, Sinha P. Myeloid-derived suppressor cells: linking inflammation and cancer. J Immunol. 2009; 182:4499-4506.

33. Curotto de Lafaille MA, Lafaille JJ. Natural and adaptive foxp3+ regulatory T cells: more of the same or a division of labor? Immunity. 2009; 30:626-635.

34. Sica A, Larghi P, Mancino A, Rubino L, Porta C, Totaro MG, Rimoldi M, Biswas SK, Allavena P, Mantovani A. Macrophage polarization in tumour progression. Semin Cancer Biol. 2008; 18:349-355.

35. Sousa S, Brion R, Lintunen M, Kronqvist P, Sandholm J, Monkkonen J, Kellokumpu-Lehtinen PL, Lauttia S, Tynninen O, Joensuu H, Heymann D, Maatta JA. Human breast cancer cells educate macrophages toward the M2 activation status. Breast Cancer Res. 2015; 17:101.

36. Kadl A, Meher AK, Sharma PR, Lee MY, Doran AC, Johnstone SR, Elliott MR, Gruber F, Han J, Chen W, Kensler T, Ravichandran KS, Isakson BE, et al. Identification of a novel macrophage phenotype that develops in response to atherogenic phospholipids via Nrf2. Circ Res. 2010; 107:737-746.

37. Balkwill F, Mantovani A. Inflammation and cancer: back to Virchow? Lancet. 2001; 357:539-545.

38. Grindel JM, Migdalof BH, Cressman WA. The comparative metabolism and disposition of penfluridol-3H in the rat, rabbit, dog, and man. Drug Metab Dispos. 1979; 7:325-329.

39. Fofaria NM, Srivastava SK. STAT3 induces anoikis resistance, promotes cell invasion and metastatic potential in pancreatic cancer cells. Carcinogenesis. 2015; 36:142-150.

40. Gupta P, Wright SE, Srivastava SK. PEITC treatment suppresses myeloid derived tumor suppressor cells to inhibit breast tumor growth. Oncoimmunology. 2015; 4:e981449. 